\title{
RECHERCHE D'UN MILIEU DE CULTURE APPROPRIÉ A LA BACTÉRIOLOGIE FROMAGËRE
}

Travail de l'Institut des Recherches Agronomiques (Station régionale de Récherches laitières de Poligny.)

par

\section{G. GUTTTONNEAU}

Directeur du Laboratoire national des Industries laitières

\section{P. SAJOUS}

Diresteur de la Station régionale de

Recherches laitières et de l'Ecole d'Industrie laitière de Poligny et

Préparateur à la Station de Poligny

Les processus microbiens dont on a à se préoccuper en bactériologie fromagère sont ceux qui interviennent lors de la préparation et de l'égouttage du eaillé ou durant sa maturation, que celle-ci soit normale ou anormale. Seul, l'examen microscopique direct peut permettre de repérer dans le milieu où ils évoluent les microorganismes actifs au cours de ces diverses phases de la fabrication et, comme dans toutes les autres branches de la microbiologie, la microscopie se trouve ici à la base des techniques de recherche.

Mais les examens microscopiques du lait, du lacto-sérum, ou de la pâtes de fromages ne sauraient, à eux seuls, permettre de résoudre tous les problèmes d'ordre bactériologique qui se posent en fromagerie.

Déjà en ce qui concerne la simple étude des proliférations microbiennes, nous savons parfaitement que les numérations microscopiques cessent d'être applicables dès qu'on se trouve en présence de milieux qui ne sont pas très richement peuplés de germes. Nous savons aussi que ces germes, pour être identitiés avec sûreté, doivent souvent être isolés à l'état de cultures pures et que, d'ailleurs, l'étude biochimique des cultures pures s'impose impérieusement dans bien des cas en microbiologie.

Et cela revient à dire que l'emploi des gelées nutritives transparentes, où toute prolifération de germes se traduit par l'apparition de colonies faciles à voir, à compter et à repiquer, répond de son côté à des besoins de la technique microbiologique que ne saurait satisfaire la microscopie.

$$
*^{*} *
$$

Encore est-il qu'il convient de n'employer les milieux nutritifs gélifiés qu'avec discernement et de ne leur demander que des indications qu'ils peuvent effectivement fournir.

De tels milieux, quelle que soit leur composition et quelles que soient les conditions dans lesquelles on les emploie, ne sauraient évidemment 
offrir des conjonctures physiologiques également fa vora bles à toutes les espèces microbiennes susceptibles de leur être incorporées au cours de recherches sans coordination. Ils sont inévitablement sélectifs et c'est précisément sur ce point qu'il faut fixer son attention lorsqu'on veut faire appel à leurs services.

Quand il s'agit de contrôles comme ceux qui sont destinés à faire connaître les qualités hygiéniques de certains produits alimentaires, la question importante est d'obtenir des résultats toujours comparables entre eux. On y parvient d'une manière satisfaisante en standardisant la composition et le mode d'emploi des milieux de culture de telle sorte que leur action sélective s'exerce toujours dans le même sens et se trouve ainsi masquée. En ce qui concerne le lait et ses dérivés, le mieux qu'il y ait à faire dans cet ordre d'idée est sans doute de se rallier aux formules qu'a proposées l'Ecole américaine (1).

Ces formules arbitrairement établies ne sauraient au contraire s'imposer lorsqu'on a en vue des recherches visant à éclairer les pratiques de l'industrie fromagère. Dans ces industries, les microorganismes n'interviennent, en effet, qu'à la faveur de conditions biologiques bien définies, en dehors desquelles leur étude perd à peu près tout intérêt. Il importe dons de ne pas s'en écarter et les meilleưrs milieux de culture utilisables en bactériologie tromagère sont par conséquent ceux qui reproduisent le plus fidèlement les conjonctures physiologiques normalement offertes aux microorganismes dans la pâte des fromages.

$$
\text { * * }
$$

La pâte des tromages contient évidemment tous les éléments simples qui entrent dans la composition du lait, puisque le caillé retient toujours une certaine proportion de lactosérum. Mais le coagulum azoté qui forme plus de la moitié de sa masse diffère de la caséine. Il en dérive par voie de dégradations diastasiques déclenchées lors de l'emprésurage et qui ne prennent fin qu'avec l'affinage.

Or, le lait écrémé soumis à des digestions diastasiques ménagées perd son opacité. Si aux produits de ces digestions on incorpore de la gélose qui ne modifie pas sensiblement leurs qualités nutritives, on obtient des gelées assez transparentes pour être d'un emploi commode en bactériologie. Ces gelées nutritives semblent, a priori, plus semblables qu'aucune autre au milieu biologique que représente la pâte des tromages. Elles ne pouvaient dès lors manquer de retenir notre attention.

Nous les a vons étudiées de près, a vec le souci de trouver la meilleure formule à adopter pour leur préparation. Ce sont les recherches ainsi entreprises que nous nous proposons de rapporter dans ce mémoire dont nous devons tout d'abord justifier le plan.

(1) Le Lait : juin, juillet, aoùt, septembre, octobre, novembre, décembre 1925 et mars 1926. 


\section{CADRE DE NOS RECHERCHES ET PLAN DE NOTRE TRAVAIL}

Les études dont il sera question par la suite ont été poursuivies à la Station de Recherches laitières de Poligny (Jura) sur les laits journellement reçus à l'Ecole d'industrie laitière annexée à la Station, en vue de la tabrication du fromage de Comté.

Avant de les soumettre à toute action diastasique, nous passions ces laits à l'écrémeuse centrifuge, en vue de les débarrasser de leur matière grasse qui ne pouvait être que gênante dans nos essais.

Les considérations mêmes que nous avons antérieurement développées nous conduisaient tout naturellement à soumettre notre lait écrémé à l'action pure et simple des extraits de présure couramment employés en fromagerie. Nous n'avons pas manqué d'examiner ce qu'il était pratiquement possible d'entreprendre dans cet ordre d'idées, mais nous nous sommes bien gardés de nous en tenir là.

La dégradation que la présure fait subir à la caséine se ramène, selon toute vraisemblance, à un phénomène de digestion pepsique et il est plus simple, au laboratoire, de conduire une telle digestion en partant directement d'extraits pepsiques. A côté des laits écrémés digérés par la présure, nous avons done mis à l'épreuvè, au point de vue de leur valeur nutritive pour les microorganismes du lait actifs en fromagerie, des laits écrémés préalablement soumis à l'action de pepsines com. merciales.

Nous n'avons pas hésité, en outre, à admettre que les polypeptides à grosse molécule qui a pparaissent dans la première phase des digestions trypsiques de la caséine peuvent avoir pour les microorganismes une valeur nutritive égale ou supérieure à celle des polypeptides résultant des digestions pepsiques de la phosphoprotéine. Nous avons done également examiné les produits obtenus en soumettant le lait écrémé à l'action ménagée de trypsines commerciales.

Sans jamais sortir du cadre de la bactériologie fromagère, nous nous sommes proposés d'établir une comparaison entre la valeur nutritive des trois types de milieux auxquels il vient d'être fait allusion, de manière à pouvoir fixer notre choix sur le meilleur.

Mais avant de nous occuper de cette comparaison décisive, nous aurons à faire connaître ce que nous ont appris les études particulières que nous avons dû entreprendre à propos de chacun des trois types de milieux envisagés. Dans cette partie de notre exposé, nous nous occuperons d'abord des digestions trypsiques et des digestions pepsiques qui sont les mieux définies au point de vue biochimique, puis des digestions par la présure, moins bien connues quant à leur mécanisme, mais en rapport plus immédiat avec les conditions de la pratique fro. magère. Cela nous permettra d'éviter des redites tout en laissant à leur place certains détails importants. 
$\mathrm{Si}$, à ce qui précède, nous ajoutons que nous avons voulu alléger nos comptes rendus d'expérience en commençant par détacher du reste de notre exposé tout ce que nous croyions a voir à dire de général au point de vue technique, il apparaît tout de suite que ce mémoire se divisera en cinq parties :

I. Données techniques sur les méthodes suivies.

II. Etude des milieux gélosés à base de lait écrémé digéré par la trypsine.

III. Etude des milieux gélosés à base de lait écrémé digéré par la pepsine.

IV. Etude des milieux gélosés à basse de lait écrémé digéré par la présure.

V. Comparaison entre les trois meilleurs milieux des types précédents.

Conclusion.

\section{DONNÉES TECHNIQUES SUR LES MÉTHODES SUIVIES}

Au cours de ce travail, nous avons fait appel à un certain nombre de techniques a pplicables à toutes nos recherches - a vec, bien entendu, les modifications de détail nécessaires pour chaque diastase - sur lesquelles nous devons donner quelques précisions. Ces techniques se rapportent : A) à l'obtention de digestions aseptiques ; B) à l'analye des liquides de digestion; C) à la préparation des divers milieux gélosés ; D) à l'essai de la valeur des milieux préparés.

\section{A. - Digestions aseptiques}

Pour que les produits de digestions obtenus a vec une même diastase fussent comparables entre eux, il était nécessaire que la diastase étudiée fût seule à agir sur le lait, indépendamment de toute action microbienne.

Il était done indispensable d'utiliser des solutions stériles de diastases et pour les obtenir on ne pouvait avoir recours qu'à la filtration sur bougie.

Las solutions diastasiques, préparées aux doses qui seront indiquées plus loin pour chacune d'elles, étaient done tiltrées sur bougie Chamberland L3 dans un appareil de Kitasato.

Le lait d'expérience stérilisé à $120^{\circ}$ dans des flacons gradués de 350 cc. (flacons pharmaceutiques) était additionné aseptiquement d'une quantité déterminée de solution diastasique stérile, puis, suivant les cas, d'une solution acide ou alcaline stérilisée.

Après agitation, les flacons étaient portés à l'étuve à $38^{\circ}$ et agités 2 ou 3 fois par jour au cours de la digestion. 


\section{B. - ANALYSE DES LAITS DIQÉRÉS}

Au sortir de l'étuve, le liquide plus ou moins louche était porté à l'autoclave à $120^{\circ}$ pendant un quart d'heure dans le but de détruire la diastase restante et de précipiter divers éléments susceptibles de troubler ultérieurement le milieu. L'eau perdue par évaporation êtait alors remplacée par de l'eau distillée et le liquide était filtré, après repos, sur papier Laurent.

Sur une partie du liquide clair, on procédait à l'analyse chimique ; le reste servait à la préparation du milieu gélosé. Les dosages suivants ont été effectués :

Acidité : Elle a été exprimée en nombre de centimètres cubes de Soude $\mathrm{N} / 10$ nécessaires pour saturer $10 \mathrm{cc}$. du liquide en présence de phénolphtaléine comme indicateur.

Acides aminés : La méthode employée pour.suivre leur apparition au cours de la digestion a été la méthode de Sörensen au formol. Le titrage était fait sur $10 \mathrm{cc}$. du liquide filtrésur pa pier et débarrassé de son azote ammoniacal. La liqueur basique employée était de la soude $\mathbf{N} / 10$. En multipliant le nombre de cc. de soude $\mathbf{N} / 10$ trouvés par 0,14 , on avait en grammes, l'azote des acides aminés titrable au formol, par litre de liquide. C'est ce chiffre qui figure dans nos tableaux. Il faut rappeler que le chiffre ainsi obtenu ne représente pas d'une façon exacte la totalité de l'azote des acides aminés. En effet : $a$ ) cette façon de calculer - la seule possible du reste - suppose qu'כ̀ un $\mathrm{NaOH}$ utilisé correspond un $\mathrm{NH}^{2}$, donc un seul $\mathrm{N}$, c'est-à-dire qu'on n'a affaire qu'à des dérivés monoazotés, alors qu'il en existe aussi de bi et de triazotés ; $b$ ) On sait que la méthode ne donne pas, avec les différents acides aminés, des résultats d'égale valeur.

Mais ces réserves faites, il est hors de doute que la méthode de Sörensen couramment employée dans l'étude des digestions trypsiques suffisait parfaitement aux besoins de nos recherches.

Azote total : Il a été obtenu en dosant par la méthode de Kjeldahl l'azote des liquides de digestion.

Azote ammoniacal : Il a été obtenu par distillation des liquides en présence de magnésie.

\section{c. - PRÉpARATION DES MiLieuX aÉlosés}

Les liquides filtrés a près chauffage à $120^{\circ}$ à l'autoclave, ainsi qu'i] a été dit plus haut, étaient dilués de manière à les amener à une même richesse en azote. A la suite les essais préliminaires qui seront décrits plus loin, cette richesse a été fixée à $1 \mathrm{gr} .8$ d'azote par litre.

Avant d'amener les liquides au volume calculé, on y ajoutait de la soude normale en s'arrêtant un peu a vant la neutralité à la phénolphta. léine et on complétait avec de l'eau distillée. On ajoutait 1,6\% de gélose qu'on laissait macérer dans le liquide pendant 1 heure ou 2 , 
puis on portait le tout au bain-marie bouillant jusqu'à dissolution complète de la gélose.

On ajustait le milieu gélosé à un $p H$ de 6,8-6,9 suivant la méthode de Barnettet Chapman(1). Cette concentrationen ions $H$, hien indiquée pour nos travaux, semble être d'ailleurs très favorable à l'étude des germes du lait. Elle est notamment recommandée dans les méthodes officielles américaines d'analyse antérieurement citées. Nous ne pouvions done hésiter à l'adopter.

Le milieu était alors porté à l'autoclave à $115^{\circ}$ pendant 15 à 20 minutes, ramené à son volume initial avec de l'eau distillée et filtré à chaud sur papier Chardin. On répartissait enfin 10 à $12 \mathrm{cc}$. de milieu gélosé dans des tubes stériles. On stérilisait une dernière fois à $110-112^{\circ}$ pendant 10 minutes et on eonservait les tubes au frais et à l'obscurité.

\section{D. - essaI de LA VALEuR des MilieuX préparés}

Pour comparer entre eux les différents milieux obtenus et déterminer quels étaient les plus favorables à la croissance des germes du lait, il n'y a vait qu'une méthode d'indiquée : faire des essais parallèles de numération sur les divers milieux, en partant d'un même lait.

Un échantillon du lait livréle matin, dans un grand état de fraîcheur, à l'Ezole d'Industrie laitière de Poligny était, a près mélange dans la chaudière, immédiatement apporté au laboratoire où plusieurs dilutions étaient préparées. Les ensemencements étaient faits le plus souvent simultanément sur les milieux essayés, ou tout au moins de manière à passer très rapidement d'un milieu à un autre. Les dilutions à $1 / 1.000$ et à $1 / 10.000$ étaient ensemencées dans trois boîtes de Pétri pour chaque milieu, de manière à obtenir un groupe de plaques avee un nombre de eolonies compris entre 30 et 300 .

Dans les essais préliminaires d'orientation, les ensemencements étaient faits en double et les boîtes de Pétri mises les unes à $30^{\circ}$, les autres à $37^{\circ}$. Comme nous le verrons, c'est la température de $30^{\circ}$ qui a été adoptée et toutes les cultures ont ensuite été faites à cette température.

Le comptage des colonies a été fait le cinquième jour de culture en utilisant une loupe de grossissement 2.5. Le nombre indiqué représente la moyenne de trois comptages effectués sur trois boites différentes. Nous avons vérifié qu'a près 7 jours, l'augmentation du nombre des colonies n'était plus sensible.

\section{ETUDE DES MILIEUX GÉLOSÉS A BASE DE LATT ÉCRÉMÉ DIGÉRÉ PAR LA TRYPSINE (2)}

Avant d'aborder plus à fond cette étude, il était indispensable de

(1) Barnetr G.D, et Chapuan H.S. : Colorimétric determination of reaction of bacteriologio mediums and other fluids. Journ. Amer. Med. Assoc. 70, 1062-1063, 1918.

(2) Trypsine Rhône-Poulenc. 
fixer par des essais préliminaires certaines conditions expérimentales : quelle devait être la teneur uniforme en azote des milieux gélosés ? Quelle était la température la plus favorable aux cultures sur agar ?

Ceci fait, il devait rester à déterminer par d'autres recherches l'influence de la quantité de trypsine, de l'alcalinité, du temps de digestion, sur la composition et la valeur nutritive du milieu obtenu, de manière à dégager les meilleures conditions à réaliser pour la préparation du milieu gélosé lait écrémé-trypsine (1).

\section{A. - ESSAIS PRÉLIMINAIRES D'ORIENTATION}

La trypsine agissant en milieu légèrement alcalin, la solution trypsique a été faite dans la soude $\mathrm{N} / 40$ à la dose de $0,5 \%$, conformément aux indications antérieurement données par l'un de nous (2).

EXPÉRIENCE 1. - Elle a été conduite comme il suit :

$100 \mathrm{cc}$. de la solution trypsique stérile ont été ajoutés à $1.000 \mathrm{cc}$. de lait stérilisé et le flacon a été maintenu à l'étuve à $38^{\circ}-40^{\circ}$, température la plus favorable à la digestion recherchée.

Le liquide est devenu progressivement translucide en prenant une teinte marron-clair. La digestion par la trypsine est assez rapide, plus rapide que la digestion par la pepsine ou par la présure. Au bout du quatrième jour, la solubilisation de la caséine était à peu près complète. Dans d'autres essais, elle a même été plus rapide encore. Le flacon n'a néanmoins été retiré de l'étuve qu'au bout du sixième jour de digestion. Le liquide dosait après stérilisation et filtration 4,74\%o d'azote solubilisé, teneur bien supérieure à celle des milieux usuels. Une dilution semblait done s'imposer.

La coloration marron devait gagner aussi à être éclaircie pour permettre un comptage plus facile des colonies. Cette dilution a été faite, soit avec de l'eau, soit avee du lactosérum que nous avons eu l'idée d'essayer parce qu'il n'introduisait dans nos milieux rien d'autre que les éléments normalement contenus dans le lait.

Nous a vons done préparé trois milieux gélosés et diversement dilués, comme il est indiqué ci-après.

Milieu I. - Obtenu en diluant le lait digéré a vec du lactosérum de fromagerie préalablement écrémé et passé à l'autoclave à $120^{\circ}$, de manière à obtenir $1,8 \%$ à $2 \%$ d'azote dans le mélange.

Milieu 2. - Obtenu en diluant le lait digéré a vec de l'eau distillée de manière à obtenir $1,8 \%$ d'azote dans le mélange.

(1) Pour abréger, nous désignerons par les expressions lait écrémé-trypsine, lait écrémépepsine, lait écrémé-présure, les produits de la digestion du lait écrémé par la trypsine, la pepsine ou la présure.

(2) G. Guitronateau : Le nettoyage et la désinfection du matériel et des locaux en industrie laitière. Journal d'Industrie laitière, août 1927. 
Milieu 3. - Obtenu en diluant le lait digéré a vec de l'eau distillée de manière à obtenir $3,5 \%$ d'azote dans le mélange.

Plusieurs essais ont été faits avec ces trois milieux suivant la technique précédemment indiquée. Les plaques d'agar correspondantes ont été maintenues d'une part à $30^{\circ}$ et d'autre part à $37^{\circ}$.

Si l'on considère la température atteinte au cours de la maturation des fromages à pâte cuite qui nous intéressent plus particulièrement ici, on doit remarquer que la température en cave chaude ne dépasse guère $28^{\circ}$. La température de $30^{\circ}$ pour les cultures semblait done la plus rationnelle dans ncs $\epsilon$ ssais. Cependant, il était intéressant de comparer aux résultats obtenus à $30^{\circ}$, ceux que donnent les cultures à $37^{\circ}$, température qui est aussi utilisée parfois et qui est recommandée par l'«Association américaine d'hygiène publique ». Les résultats obtenus sont consignés dans le tableau $\mathrm{I}$.

\section{TABLEAU I}

RECHERCHES PRÉLIMINATRES SUR LES MILIEUX LAIT ÉCRÉMÉ - TRYPSINE (Comptage des colonies le $5^{\ominus}$ jour).

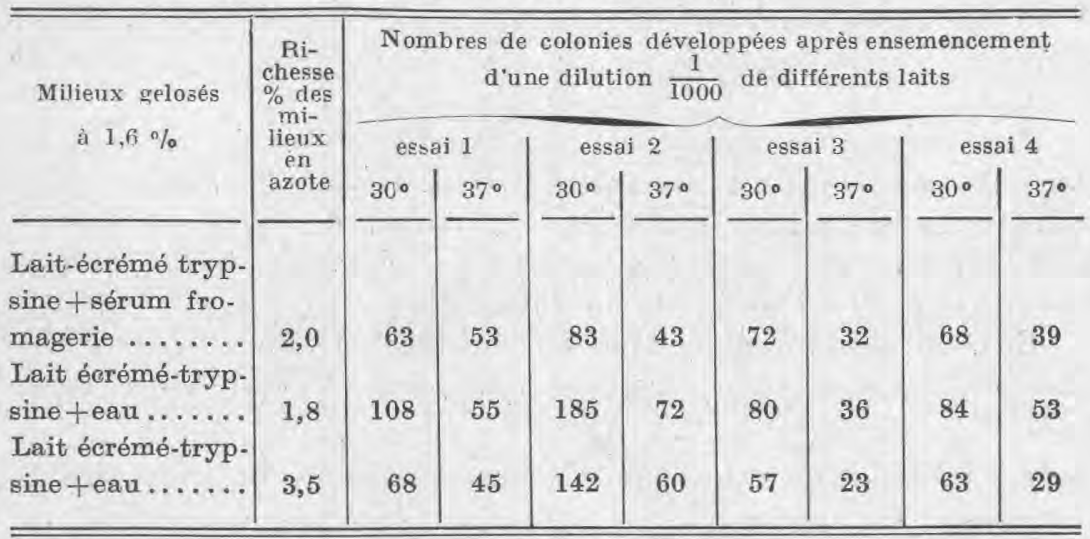

Interprétation des résultats de l'expérience $I$. - Le tableau I montre clairement :

$1^{0}$ Qu'une dose trop forte d'azote nuit au développement d'un nombre relativement important de colonies, les chiffres du milieu 2 étant constamment et sensiblement supérieurs à ceux du milieu 3.

$2^{\circ}$ Que pour effectuer les dilutions, l'eau donne des résultats supérieurs à ceux obtenus avec le sérum de fromagerie.

$3^{\circ}$ Que la température de $30^{\circ}$ est plus favorable que la température de $37^{\circ}$.

Les essais préliminaires que nous venons de résumer nous ont done conduit à choisir pour tous les essais qui vont suivre : $a$ ) Une richesse en azote soluble de $1,8 \%$; $b$ ) L'eau distillée pour diluer les laits digérés; c) Une température d'incubation de $30^{\circ}$. 


\section{B. - influence sur la composition du Milieu, De LA dose DE TRYPSINE EMPLOYEE, DE L'ALOALINITE A L'ORIGINE, ET DU TEMPS DE DIGESTION}

Nous savons que la valeur nutritive des milieux à base de lait digéré peut être influencée par l'état de dégradation de la caséine après digestion et cet état dépend notamment de la dose de diastase employée, de la réaction du milieu au cours de la digestion et du temps que dure celle-ci. Ces diverses influences ont été étudiées dans les expériences II et III.

EXPÉRIence II. - En ce qui concerne la proportion de soude employée, le liquide s'acidifiant au fur et à mesure de la solubilisation de la caséine, nous avons cherché à obtenir en fin de digestion un liquide peu acide et nous avons ainsi été conduits à essayer la soude $\mathrm{N} / 40$ et la soude $\mathrm{N} / 10$ dans la préparation des solutions trypsiques.

En ce qui concerne la dose de diastase, comme la trypsine commerciale dont nous disposions n'entrait pas totalement en solution à la concentration de $1 \%$, nous avons dû nous en tenir aux solutions à $0,5 \%$ et nous avons ajouté au lait $10 \%$ ou $20 \%$ de cette solution trypsique (correspondant à 0 gr. 5 ou à 1 gr. de trypsine par litre de lait écrémé).

Pour déterminer l'influenze du temps de digestion, nous avons poursuivi chaque série d'essais sur 4 flacons. Tous les deux jours, c'està-dire au bout de $2,4,6$ et 8 jours, un flacon de chaque série était retiré de l'étu ve et stérilisé. Une partie de son contenu servait à l'analyse et a vec le reste on préparait un milieu gélosé.

En résumé, pour tenir compte des divers facteurs dont il vient d'être question, nos essais ont été répartis comme suit en quatre séries parallèles :

Série $\mathrm{I}-100 \mathrm{cc}$. de solution à $5 \%$ dans la soude $\mathrm{N} / 40$ pour $1.000 \mathrm{cc}$. de lait -4 flacons.

Série II -200 cc. de solution à $5 \%$ dans la soude $\mathrm{N} / 40$ pour $1.000 \mathrm{cc}$. de lait -4 flacons.

Série 1 II $-100 \mathrm{cc}$. de solution à $5 \%$ o dans la soude $\mathrm{N} / 10$ pour $1.000 \mathrm{cc}$. de lait -4 flacons.

Série IV $-200 \mathrm{co}$. de solution à $5 \%$ dans la soude $\mathrm{N} / 10$ pour $1.000 \mathrm{ce}$. de lait -4 flacons.

Pour plus de commodité, ces essais ont été faits sur $200 \mathrm{cc}$. de lait contenus, comme il a été dit, dans des flacons gradués de 350 cc. Les 16 flacons ont été préparés en même temps avec un même lait et mis à l'étu ve à $38-40^{\circ}$.

Disons tout de suite que les laits des séries III et IV ne présentaient aucune trace de digestion au bout de 8 jours d'étuve alors que tous les autres laits avaient déjà pris une teinte brune en 24 heures. Dans un 
nouvel essai effectué dans les mêmes conditions que le précédent, nous avons constaté qu'après 12 jours d'étuve il n'y avait pas encore a pparence de digestion de la caséine. Il fallait donc reconnaître que, dans les conditions de nos expériences, la soude $\mathrm{N} / 10$ employée pour la préparation des solutions de trypsine détruisait la diastase ou paralysisait son action.

Dans le tableau II figurent seuls, par conséquent, les résultats obtenus a vec les séries I et II.

Faisons observer que notre solution de trypsine dans la soude $\mathrm{N} / 40$ était légèrement ammoniacale, probablement par suite du mode de préparation de la diastase. La teneur en azote ammoniacal était de 0 gr. 280 par litre de solution a près filtration sur bougie. Nous avons fait, à cet égard, toutes les corrections qui s'imposaient.

Interprétation des résultats de l'expérience II. - L'examen du tableau II montre que, dans chaque série, l'azote total du liquide digéré ne varie pour ainsi dire pas a près le deuxième jour de digestion. C'est dans la série II a vec $200 \mathrm{cc}$. de solution trypsique que le taux d'azote soluble est le plus élevé ; il atteint 5 gr. 47 alors que le lait contenait 5 gr. 67 d'azote total, e'est-à-dire que l'azote du liquide digéré représente $96,5 \%$ de l'azote total du lait.

L'azote des acides aminés augmente régulièrement du début à la fin de la digestion. Le chiffre d'azote ammoniacal est également un peu plus élevé à la fin qu'au début. C'est d'ailleurs la dose de 1 gramme de trypsine par litre de lait qui a produit au bout de 8 jours la digestion la plus complète. Ces trois faits sont dans l'ordre de nos connaissances générales sur la marche des digestions trypsiques.

Il est à peine besoin de rappeler que les trypsines commerciales, comme les pepsines du reste, n'ont pas une action diastasique constante, pour un poids donné. Suivant leur origine et leur mode de préparation, elles peuvent produire des digestions plus ou moins rapides.

\section{c. - RECHERCHE D'UN MILIEU RESTANT ALCALIN PENDANT TOUTE LA DURÉE DE LA DIGESTION}

Dans l'expérience II le liquide était toujours acide en fin de digestion. Nous avons cherché à obtenir un milieu restant constamment alcalin, mais présentant seulement une alcalinité faible à la tin de la digestion.

EXPÉRIENCE II (bis). - Après quelques tâtonnements, nous a vons fixé à $50 \mathrm{cc}$. de soude normale la dose de soude à ajouter par litre de lait, à l'origine, pour arriver à ce résultat. Nous avons conservé le même dispositif expérimental que pour l'expérience II, série II (200 cc. de solution trypsique à $0,50 \%$ par litre de lait) en ajoutant en outre 
TABLEAU II

DIGESTION DU LAIT ECREME PAR LA TRYPSINE

\begin{tabular}{|c|c|c|c|c|c|c|c|c|c|c|c|c|}
\hline \multirow{2}{*}{ 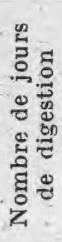 } & \multirow{2}{*}{ 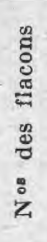 } & \multicolumn{2}{|c|}{$\begin{array}{l}\text { Pour } 1000 \text { ce. } \\
\text { de lait écrémé }\end{array}$} & \multirow{2}{*}{ 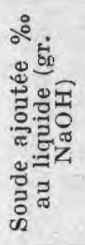 } & \multirow[b]{2}{*}{$\begin{array}{l}\text { Volume inal en cc. } \\
\begin{array}{c}\text { du iqquide soumis } \\
\text { ने la digestion }\end{array}\end{array}$} & \multicolumn{4}{|c|}{ Analyse chimique du liquide de digeslion } & \multicolumn{3}{|c|}{$\begin{array}{c}\text { Chiffres rapportés à } 1000 \text { cc. } \\
\text { de lait écrémé }\end{array}$} \\
\hline & & $\begin{array}{c}\text { Quantité } \\
\text { de } \\
\text { trypsine }\end{array}$ & $\begin{array}{l}\text { Addition de } \\
\text { solution } \\
\text { trypsique à } \\
0,5 \% \text { dans } \\
\text { soude N / } 40\end{array}$ & & & $\begin{array}{l}\text { A cidité } \\
\text { (cc. soude } \\
\text { N/1.0 pour } \\
10 \text { cc.) }\end{array}$ & $\begin{array}{l}\text { Azote } \\
\text { total en } \\
\text { gr. } \% \text {. }\end{array}$ & $\begin{array}{c}\text { Azote } \\
\text { des acides } \\
\text { aminés } \\
\text { titrable } \\
\text { au formol } \\
\text { en gr } \% \circ\end{array}$ & $\begin{array}{l}\text { Azote } \\
\text { ammo- } \\
\text { niacal en } \\
\text { gr. \%o }\end{array}$ & $\begin{array}{l}\text { Azote } \\
\text { total } \\
\text { en gr. }\end{array}$ & $\begin{array}{c}\text { Azote } \\
\text { des acides } \\
\text { aminés } \\
\text { titrable } \\
\text { au formol } \\
\text { en gr. }\end{array}$ & $\begin{array}{l}\text { Azote } \\
\text { ammo- } \\
\text { niacal } \\
\text { en gr. }\end{array}$ \\
\hline 2 & 1 & série I & & 0,09 & 1100 & 3,4 & 4,84 & 0,543 & 0,160 & 5,32 & 0,597 & 0,176 \\
\hline 4 & 2 & & & 0,09 & 1100 & 3,1 & 4,89 & 0,622 & 0,165 & 5,38 & 0,684 & 0,181 \\
\hline 6 & 3 & & & 0,09 & 1100 & $3 ; 3$ & 4,86 & 0,678 & 0,165 & 5,34 & 0,745 & 0,181 \\
\hline 8 & 4 & & 1 & 0,09 & 1100 & 3,0 & 4,86 & 0,693 & 0,192 & 5,34 & 0,761 & 0,211 \\
\hline 2 & 5 & & & 0,166 & 1200 & 3,2 & 4,56 & 0,606 & 0,174 & 5,47 & 0,727 & 0,209 \\
\hline 4 & 6 & série & & 0,166 & 1200 & 2,9 & 4,54 & 0,706 & 0,162 & 5,44 & 0,847 & 0,194 \\
\hline 6 & 7 & $1 \mathrm{gr}$. & & 0,166 & 1200 & 3,0 & 4,56 & 0,700 & 0,179 & 5,47 & 0,840 & 0,215 \\
\hline 8 & 8 & & & 0,166 & 1200 & 3,0 & 4,56 & 0,714 & 0,179 & 5,47 & 0,857 & 0,215 \\
\hline
\end{tabular}

Composition du lait écrémé d'expérience
Acidité . . . . . I ce. 9 soude N/10 pour 10 ec.

Lactose. . . . . . 49,8 gr. par litre.

Azote total ..... 5, 5,

Azote titrable au formol 0,090 ,

Azote ammoniacal.... $0,059 \quad$ n 


\section{TABLEAU II bis}

DIGESTION DU LAIT ÉCRÉMÉ PAR LA TRYPSINE EN MILIEU MAINTENU CONSTAMMENT ALCALIN

\begin{tabular}{|c|c|c|c|c|c|c|c|c|c|c|c|c|c|}
\hline \multirow{2}{*}{ 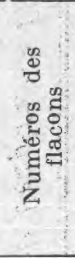 } & \multirow{2}{*}{ 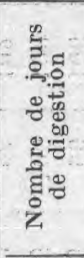 } & \multicolumn{3}{|c|}{ Pour $100 \mathrm{cc}$ de lait écrémé } & \multirow{2}{*}{ 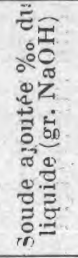 } & \multirow{2}{*}{ 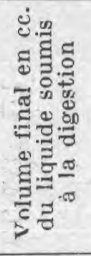 } & \multicolumn{4}{|c|}{ Analyse chimique du liquide de digestion } & \multicolumn{3}{|c|}{$\begin{array}{c}\text { Chiffres rapportés à } 1.000 \text { cc. } \\
\text { de lait écrémé }\end{array}$} \\
\hline & & $\begin{array}{c}\text { Quantité } \\
\text { de } \\
\text { trypsine }\end{array}$ & $\begin{array}{c}\text { Addition } \\
\text { de solution } \\
\text { trypsique à } \\
0,50 \text { dans } \\
\text { soude } \mathrm{N} / 40\end{array}$ & $\begin{array}{c}\begin{array}{c}\text { Addition } \\
\text { de } \\
\text { Soude N }\end{array} \\
\end{array}$ & & & $\begin{array}{c}\text { Alcalinité } \\
\text { (cc. Acide } \\
\text { lactique } \\
\mathrm{N} / 10 \\
\text { pour } 10 \text { cc.) }\end{array}$ & $\begin{array}{l}\text { Azote } \\
\text { total en } \\
\text { gr. } \% \text { o }\end{array}$ & $\begin{array}{c}\text { Azote } \\
\text { des acides } \\
\text { aminés } \\
\text { titrahle } \\
\text { au formol } \\
\text { en gr. \%o }\end{array}$ & $\begin{array}{c}\text { Azote } \\
\text { ammo- } \\
\text { niacal } \\
\text { en gr. \%o }\end{array}$ & $\begin{array}{l}\text { Azote } \\
\text { total } \\
\text { en gr. }\end{array}$ & $\begin{array}{c}\text { Azote } \\
\text { des acides } \\
\text { aminés } \\
\text { titrable } \\
\text { au formol } \\
\text { en gr. }\end{array}$ & $\begin{array}{l}\text { Azote } \\
\text { ammo- } \\
\text { niacal } \\
\text { en gr. }\end{array}$ \\
\hline 9 & 4 & & I? & & & & 0,5 & 4,32 & 0,636 & 0,146 & 5,4 & 0,79 & 0,182 \\
\hline 10 & 6 & $1 \mathrm{gr}$, & $200 \mathrm{cc}$, & $50 \mathrm{cc}$, & 1,76 & 1250 & 0,4 & 4,37 & 0,620 & 0,160 & 5,46 & $\quad 0,77$ & $0 ; 2$ \\
\hline 11 & 8 & $\cdots \because \cdots$ & 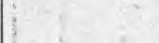 & & & & 0,4 & 4,37 & 0,650 & 0,160 & 5,46 & 0,81 & 0,2 \\
\hline
\end{tabular}

Même composition du lait écrémé que dans le tableau II.

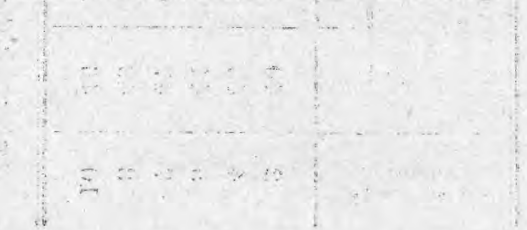


au début $50 \mathrm{cc}$. de soude normale. L'alcalinité finale a été titrée avec l'acide lactique $\mathrm{N} / 10$.

Les résultats de cette expérience figurent dans le tableau IIbis. On y verra que les chiffres obtenus pour l'azote titrable au tormol, l'azote total et l'azote ammoniacal sont très voisins, une fois rapportés à $1.000 \mathrm{cc}$. de lait, de ceux obtenus pour la série Il du tableau 11 .

\section{D. - ESSAIS DE NUMÉRATIONS SUR LES DIVERS MILIEUX OBTENUS}

EXPÉRIENCE III. - Des milieux gélosés ont été préparés avec les liquides de digestion No 2 , No 4 , No 5 , No 7 , No 8 et No 10 (voir tableaux II et II bis) suivant la technique décrite précédemment.

En ce qui concerne les milieux légèrement alcalins en fin de digestion (comme le $\mathrm{N}^{\circ} 10$ ) nous avons constaté qu'ils restaient troubles a près passage à l'autoclave à $120^{\circ}$. Pour obtenir un milieu facile à clarifier par filtration, il suffit de neutraliser avant chauffage avec une solution d'acide lactique en poussant jusqu'à réaction très légèrement acide. On amène ensuite au $p \mathrm{H}$ voulu, a près addition d'agar, comme pour les autres milieux.

Les résultats de l'expérience III sont rassemblés dans le tableau III. Afin de pouvoir comparer plus facilement entre eux les nombres de colonies trouvés pour chaque essai sur les divers milieux, les chiffres des numérations obtenus sur le milieu $\mathrm{N}^{\circ} 8$ ont été supposés égaux à 100 pour chaque essai et les autres chiffres ont été calculés proportionnellement (colonnes $6,7,8$ et 9 ). La moyenne pour chaque milieu est la moyenne des colonnes $6,7,8$ et 9 .

\section{TABLEAU III}

ESSATS DE NUMŔRATIONS DES DIFHARENTS MUIEUX GELOSfS A BASE DE LATT GORGMTE DIGERE PAR LAA TRYPSINE

(Température d'incubation : $30^{\circ}$

Comptage des colonies : le $5^{\text {e }}$ jour).

\begin{tabular}{|c|c|c|c|c|c|c|c|c|c|}
\hline \multirow{2}{*}{ 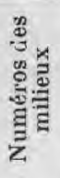 } & \multicolumn{4}{|c|}{$\begin{array}{c}\text { Nombres de colonies développées } \\
\text { après ensemencement par } 1 \text { ec. } \\
\text { d'une dilution de lait à } \frac{1}{1000} \\
\text { essais }\end{array}$} & & $\begin{array}{l}\text { bres } \\
\text { com }\end{array}$ & $\begin{array}{l}\text { olonie } \\
\text { s sur }\end{array}$ & $\begin{array}{l}\text { appor } \\
\text { milie }\end{array}$ & $\begin{array}{l}8 \text { à } 100 \\
\text { Moyenne } \\
\text { (chiffres } \\
\text { des } 4\end{array}$ \\
\hline & 1 & 2 & 3 & 4 & 1 & 2 & 3 & 4 & colonnes) \\
\hline 2 & 40 & 175 & 72 & " & 82 & 87 & .73 & " & 81 \\
\hline 4. & 43 & 200 & 78 & 57 & 88 & 100 & 79 & 88 & 89 \\
\hline 5 & 44 & 190 & 94 & 55 & 90 & 95 & 95 & 85 & 91 \\
\hline 7 & 47 & 212 & 98 & 63 & 96 & 106 & 99 & 97 & 99,5 \\
\hline 8 & 49 & 201 & 99 & 65 & 100 & 100 & 100 & 100 & 100 \\
\hline 10 & 46 & 204 & 93 & 60 & 94 & 102 & 94 & 92 & 95,5 \\
\hline
\end{tabular}

Interprétation des résultats de l'expérience III. - Sauf le No 2 , ces 
milieux ne présentent pas entre eux de grands écarts au point de vue de leur valeur nutritive. Cependant, on peut constater que ce sont les milieux 7 et 8 qui ont donné les meilleurs résultats.

\title{
E. - CONCLUSION DE NOTRE Étude SUR LES MILIEUX LAIT ECREME-TRYPSINE
}

Il ressort nettement des faits ci-dessus exposés : $1^{\circ}$ Qu'il est inutile de chercher à a voir un milieu encore alcalin en fin de digestion ; $2^{\circ} \mathrm{Qu}$ 'il est indiqué d'employer une dose de 1 gramme de trypsine par litre plutôt qu'une dose moindre ; $3^{\circ}$ Qu'il suffit de laisser la digestion se poursuivre pendant 6 jours, comme dans le milieu No 8 (Tableau II). On peut donc résumer comme suit la préparation du meilleur milieu lait écrémé-trypsine :

Ajouter à un litre de lait écrémé stérilisé $200 \mathrm{cc}$. d'une solution stérile de trypsine à $5 \%$ dans la soude à $1 \%$. Maintenir à l'étuve à $38^{\circ}$ et agiter 2 ou 3 fois par jour. Au bout de 6 jours porter à $120^{\circ}$ pour détruire la diastase et clarifier, filtrer. Ramener à une très faible acidité et diluer jusqu'à teneur en azote de 1,8\%o. Géloser à $16 \%$, ajuster à $p H$ 6,8-6,9, clarifier par chauffage à $115^{\circ}$ et filtration, répartir en tubes stériles et porter 10 minutes à $110-112^{\circ}$. (Pour les détails, voir p. 620 et 622.)

C'est un milieu digéré et préparé dans ces conditions que nous prendrons comme milieu-type : lait écrémé-trypsine.

(A suivre).

\section{SUR LES FLORES BACTÉRIENNE ET FONGIQUE DES CAILLÉS DE LAIT}

\author{
RÔLE DES SYMBIOSES DANS LA MATURATION \\ DU CAMEMBERT
}

\author{
par \\ Mademoiselle FRANÇOISE SANSONETTI \\ Doeteur en Pharmacie
}

$\mathrm{Au}$ moment des découvertes pastoriennes sur l'origine vitale des fermentations, les connaissances sur le lait étaient d'ordre purement chimique. Les méthodes analytiques avaient permis de séparer et de doser ses principaux constituants. En 1840, QUÉvENNE isolait la caséine, puis Mulder, Denis, Lteberkühn, Mrllon et Commatlle, HamMARSTEN cherchaient à en préciser la nature et les propriétés chimiques. Chevreutl et Bromeis s'efforçaient de fixer la composition du beurre. Le lactose, la caséine, le beurre étaient ainsi étudiés séparément comme des corps simples. Mais la plus grande incertitude régnait sur la cause et la nature des transformations que ces mêmes éléments subissent dans le caillé, au cours de la maturation. La pratique fro- 